Journal of Advanced Research in Fluid Mechanics and Thermal Sciences

\title{
MHD Stagnation Point Flow and Heat Transfer Over a Stretching Sheet in a Blood-Based Casson Ferrofluid With Newtonian Heating
}

\author{
Muhammad Khairul Anuar Mohamed ${ }^{1,}{ }^{\star}$, Siti Hanani Mat Yasin ${ }^{1}$, Mohd Zuki Salleh ${ }^{1}$, Hamzeh Taha \\ Alkasasbeh $^{2}$ \\ 1 Centre for Mathematical Sciences, College of Computing \& Applied Sciences, Universiti Malaysia Pahang, 26300 Gambang, Pahang, Malaysia \\ Department of Mathematics, Faculty of Science, Ajloun National University, P.O. Box 43, Ajloun 26810, Jordan
}

\section{ARTICLE INFO}

\section{Article history:}

Received 26 October 2020 Received in revised form 28 January 2021 Accepted 10 February 2021 Available online 7 April 2021

Keywords:

Casson ferrofluid; heat transfer; MHD stagnation point; stretching sheet; Newtonian heating

\section{ABSTRACT}

\begin{abstract}
The present study investigated the magnetohydrodynamic (MHD) flow and heat transfer on a stagnation point past a stretching sheet in a blood-based Casson ferrofluid with Newtonian heating boundary conditions. The ferrite $\mathrm{Fe}_{3} \mathrm{O}_{4}$ and cobalt ferrite $\mathrm{CoFe}_{2} \mathrm{O}_{4}$ ferroparticles suspended into Casson fluid represent by human blood to form blood-based Casson ferrofluid are numerically examined. The mathematical model for Casson ferrofluid which is in non-linear partial differential equations are first transformed to a more convenient form by similarity transformation approach then solved numerically by using the Runge-Kutta-Fehlberg (RKF45) method. The characteristics and effects of the stretching parameter, the magnetic parameter, the Casson parameter and the ferroparticle volume fraction for $\mathrm{Fe}_{3} \mathrm{O}_{4}$ and $\mathrm{CoFe}_{2} \mathrm{O}_{4}$ on the variation of surface temperature and the reduced skin friction coefficient are analyzed and discussed. It is found that the blood-based Casson ferrofluid provided up to $46 \%$ higher in temperature surface compared to blood-based fluid with the presence of magnetic effects.
\end{abstract}

\section{Introduction}

Study on the heat transfer over a stretching sheet is important due to its numerous industrial applications in manufacturing and process engineering such that in paper and fibres production, glass blowing, continuous casting, as well as sheeting material through extrusion process like polymer extrusion and aerodynamic extrusion [1].

Since the pioneering works of Crane [2], many investigations regarding the study on stretching sheet have been made with the extension to other effects like magnetohydrodynamic effect, thermal radiation, viscous dissipation, suction and blowing effect, slip effect, chemical reaction, heat generation, porous effects etc [3-8]. This topic becoming more attractive year by years with the introduction of numerous industrial fluid such as nanofluid, hybrid nanofluid, ferrofluid, as well as other non-Newtonian fluid like viscoelastic fluid, Jeffrey fluid and Casson fluid [9-14].

\footnotetext{
* Corresponding author.

E-mail address: mkhairulanuar@ump.edu.my
}

https://doi.org/10.37934/arfmts.82.1.111 
The flow of non-Newtonian fluids shows different characteristics compared to a Newtonian fluid like water. The usual Navier-Stokes equations did not fulfil the characteristics presented by the fluid. Therefore, some modifications to the Navier-Stokes equations are proposed in many previous studies [15]. Casson fluid model is one of the non-Newtonian model introduced to characterized the fluid elastic solid behaviour. This model is identified as the most preferred rheological model for describing human blood flow [16].

On the other hand, the flow of magnetohydrodynamic (MHD) plays an important role in medicine. It is employed in cancer treatment, reducing bleeding in severe injuries, magnetic resonance imaging and other diagnostic tests [17]. This type of fluid coin as ferrofluid. Ferrofluid containing engineered colloidal suspensions of magnetic nanoparticles like cobalt, magnetite and ferrite scattered based fluid like water and oil.

Ferrofluid was invented by NASA as liquid rocket fuel at no gravity situation [18]. Today, ferrofluid played an important role in medicine and electrical devices like heat controlling agents in electric motor, $\mathrm{Hi}-\mathrm{Fi}$ speakers as well as computer hard disc [19].

Motivated from the above literature, the study on blood-based fluid blended with magnetic nanoparticles to formed a ferrofluid on a stagnation flow with stretching surface is an interesting topic to consider. The Casson non-Newtonian fluid model combines with Tiwari and Das nanofluid model [20] is numerically examined. The realistic Newtonian heating boundary conditions is applied instead the common constant wall temperature boundary conditions. To the best of the author's knowledge, this study had never been discussed before, thus the results published here is new.

\section{Mathematical Formulations}

A steady two-dimensional stagnation point flow over a stretching sheet (Figure 1) immersed in Casson ferrofluid with ambient temperature, $T_{\infty}$. Assuming that $u$ and $v$ are the velocity components along the $x$ and $y$ axes, respectively. Next, the stretching velocity $u_{w}(x)=a x$ and the free stream velocity $U_{\infty}=b x$ are assumed in linear forms where $a$ and $b$ are positive constants [21]. Further, the stretching sheet is subjected to a Newtonian heating boundary condition as proposed by Merkin [22]. The Navier-Stokes equations can be governed as follows

$$
\frac{\partial u}{\partial x}+\frac{\partial v}{\partial y}=0
$$

$u \frac{\partial u}{\partial x}+v \frac{\partial u}{\partial y}=U_{\infty} \frac{d U_{\infty}}{d x}+v_{f f}\left(1+\frac{1}{\beta}\right) \frac{\partial^{2} u}{\partial y^{2}}-\frac{\sigma_{f f} B_{o}^{2}}{\rho_{f f}}\left(u-U_{\infty}\right)$

$u \frac{\partial T}{\partial x}+v \frac{\partial T}{\partial y}=\frac{k_{f f}}{\left(\rho C_{p}\right)_{f f}} \frac{\partial^{2} T}{\partial y^{2}}$

with boundary conditions

$$
u=u_{w}, v=0, \frac{\partial T}{\partial y}=-h_{s} T \text { at } y=0
$$


$u=U_{\infty}, T \rightarrow T_{\infty}$ as $y \rightarrow \infty$.

The Casson ferrofluid kinematic viscosity, dynamic viscosity, electrical conductivity and its density is denoted as $v_{f f}, \mu_{f f}, \sigma_{f f}$, and $\rho_{f f}$, respectively. $\beta$ and $B_{o}$ is the Casson parameter and magnetic field strength while $T$ is the temperature of the Casson ferrofluid inside the boundary layer. $\left(\rho C_{p}\right)_{f f}$ is the heat capacity of hybrid nanofluid and $k_{f f}$ is the thermal conductivity of hybrid nanofluid. Other properties related to base fluid (blood) and the ferroparticles are denoted with subscript ${ }_{f}$ and , respectively as follows [23]

$$
\begin{aligned}
& v_{f f}=\frac{\mu_{f f}}{\rho_{f f}}, \quad \rho_{f f}=(1-\phi) \rho_{f}+\phi \rho_{s}, \quad \alpha_{f f}=\frac{k_{f f}}{\left(\rho C_{p}\right)_{f f}}, \quad \mu_{f f}=\frac{\mu_{f}}{(1-\phi)^{2.5}}, \\
& \left(\rho C_{p}\right)_{f f}=(1-\phi)\left(\rho C_{p}\right)_{f}+\phi\left(\rho C_{p}\right)_{s}, \quad \frac{k_{f f}}{k_{f}}=\frac{k_{s}+2 k_{f}-2 \phi\left(k_{f}-k_{s}\right)}{k_{s}+2 k_{f}+\phi\left(k_{f}-k_{s}\right)} .
\end{aligned}
$$

where $\phi$ are ferroparticles volume fraction. Noticed that the Eq. (1)-(3) are non-linear partial differential equations which consist many dependent and independent variables. It is also in dimensional forms which is difficult to solve directly. Therefore, the similarity transformation approach is applied [23]

$\eta=\left(\frac{a}{v_{f}}\right)^{1 / 2} y, \quad \psi=\left(a v_{f}\right)^{1 / 2} x f(\eta), \quad \theta(\eta)=\frac{T-T_{\infty}}{T_{\infty}}$

Eq. (6) shows the similarity variables where $\eta, \psi$ and $\theta$ is a non-dimensional variable, stream function and temperature, respectively. The similarity variables (6) satisfy the continuity Eq. (1) by definition

$$
u=\frac{\partial \psi}{\partial y} \text { and } v=-\frac{\partial \psi}{\partial x}
$$

Next, substitute the similarity variables Eq. (6) and (7) into governing equations Eq. (2) and (3) gives the following transformed ordinary differential equations

$$
\begin{aligned}
& \frac{1}{(1-\phi)^{2.5}\left[1-\phi+\left(\phi \rho_{s}\right) /\left(\rho_{f}\right)\right]}\left(1+\frac{1}{\beta}\right) f^{\prime \prime \prime}+f f^{\prime \prime}-f^{\prime 2}+\varepsilon^{2}-M\left(f^{\prime}-\varepsilon\right)=0, \\
& \frac{1}{\operatorname{Pr}} \frac{k_{n f} / k_{f}}{(1-\phi)+\phi\left(\rho C_{p}\right)_{s} /\left(\rho C_{p}\right)_{f}} \theta^{\prime \prime}+f \theta^{\prime}=0 .
\end{aligned}
$$

The boundary conditions become

$$
f(0)=0, f^{\prime}(0)=1, \theta^{\prime}(0)=-\gamma(1+\theta(0)),
$$


$f^{\prime}(\eta) \rightarrow \varepsilon, \theta(\eta) \rightarrow 0$, as $y \rightarrow \infty$.

By definition, $M=\frac{\sigma_{f f} B_{o}^{2}(x)}{a \rho_{f f}}$ is the magnetic parameter, $\operatorname{Pr}=\frac{v_{f}\left(\rho C_{p}\right)_{f}}{k_{f}}$ is Prandtl number which will be set as 21 in calculation with respect to blood [16] $, \varepsilon=\frac{b}{a},(\varepsilon>0)$ is a stretching parameter and $\gamma=h_{s}\left(\frac{a}{v_{f}}\right)^{-1 / 2}$ is a conjugate parameter. The physical quantities interested are the surface temperature $\theta(0)$, the heat transfer rate $-\theta^{\prime}(0)$ and the skin friction coefficient $C_{f}$ which given by

$C_{f}=\frac{\tau_{w}}{\rho_{f} U_{\infty}^{2}}$,

and reduced by Bhattacharyya [24] as

$C_{f} \operatorname{Re}_{x}^{1 / 2}=\left(1+\frac{1}{\beta}\right) \frac{f^{\prime \prime}(0)}{(1-\phi)^{2.5}}$,

where $\operatorname{Re}_{x}=\frac{U_{\infty} x}{v_{f}}$ is the Reynolds number.

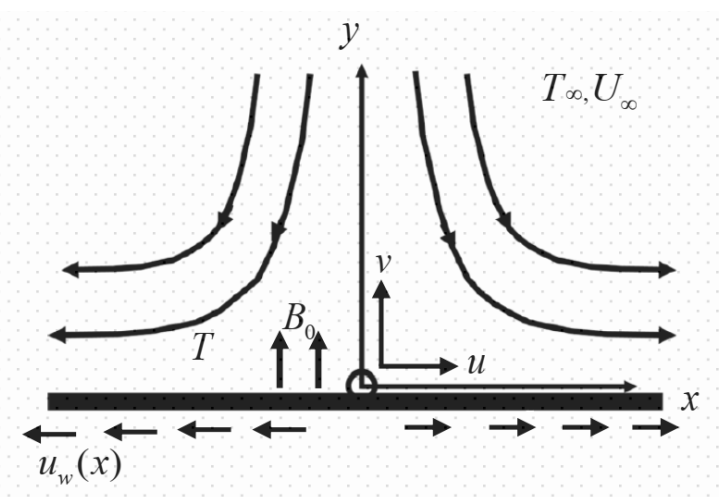

Fig. 1. Physical model and the coordinate system

\section{Results and Discussion}

The Runge-Kutta-Fehlberg (RKF45) are applied in solving the system of ordinary differential equations Eq. (8) and (9) with boundary conditions (10). The numerical results obtained for the surface temperature $\theta(0)$ and the reduced skin friction coefficient $C_{f} \operatorname{Re}_{x}^{1 / 2}$ for a various values of stretching parameter $\varepsilon$, magnetic parameter $M$, Casson parameter $\beta$ and the ferroparticle volume fraction $\phi$ for ferrite $\mathrm{Fe}_{3} \mathrm{O}_{4}$ and cobalt ferrite $\mathrm{CoFe}_{2} \mathrm{O}_{4}$. It is worth mentioning that the boundary layer thickness from 4 to 8 is sufficient to provide the accurate numerical results. Besides, the 
values of thermophysical properties of blood as a based-fluid with the ferroparticles consider are tabulated in Table 1.

Table 1

Thermophysical properties of water and ferroparticles [16]

\begin{tabular}{llll}
\hline Physical Properties & Blood $(f)$ & $\mathrm{Fe}_{3} \mathrm{O}_{4}$ & $\mathrm{CoFe}_{2} \mathrm{O}_{4}$ \\
\hline$\rho\left(\mathrm{kg} / \mathrm{m}^{3}\right)$ & 1053 & 5180 & 4907 \\
$C_{p}(\mathrm{~J} / \mathrm{kg} \cdot \mathrm{K})$ & 3594 & 670 & 700 \\
$k(\mathrm{~W} / \mathrm{m} \cdot \mathrm{K})$ & 0.492 & 9.7 & 3.7 \\
\hline
\end{tabular}

For comparison purpose, Table 2 is tabulated. It is shown that the comparison values of $f^{\prime \prime}(0)$ with previous results by Nazar et al., [25] and Bhattacharyya [24]. It is found that the numerical results are in good agreement.

Table 2

\begin{tabular}{llll}
\multicolumn{4}{l}{ Comparison values of $f^{\prime \prime}(0)$ for some values of $\varepsilon$ when $\operatorname{Pr}=7, \gamma=1, M=\phi=0$ and $\beta=\infty$} \\
\hline$\varepsilon$ & Nazar et al., [25] & Bhattacharyya [24] & Present \\
\hline 0.1 & -0.9694 & -0.969386 & -0.969395 \\
0.2 & -0.9181 & -0.918107 & -0.918108 \\
0.3 & -0.6673 & -0.667263 & -0.667264 \\
2 & 2.0176 & 2.017503 & 2.017503 \\
3 & 4.7296 & 4.729284 & 4.729282 \\
\hline
\end{tabular}

Figure 2 shows the variation of the surface temperature $\theta(0)$ for various values of the stretching parameter $\varepsilon$. It is found that the values of $\theta(0)$ is decreasing with the increase of $\varepsilon$. From Figure 2, it is observed that as $\varepsilon$ is absent $(\varepsilon=0)$, the blood-based Casson ferrofluid with cobalt and ferrite provided $17.1 \%$ and $21.4 \%$ higher in $\theta(0)$ values compared to the blood-based fluid. As $\varepsilon$ increases, the temperature difference between blood-based Casson ferrofluid and its based fluid decreases. This is a physical sign that the high value of $\varepsilon$ will fully eliminate the effects of ferroparticles on temperature in Casson ferrofluid.

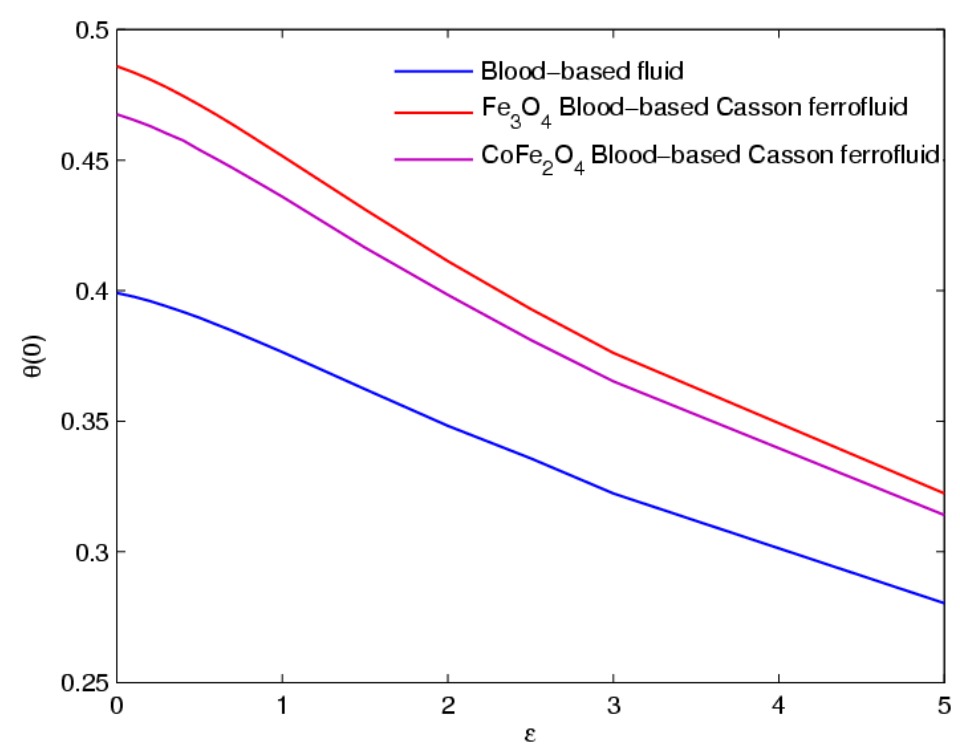

Fig. 2. Variation of $\theta(0)$ with various values of $\varepsilon$ when $\operatorname{Pr}=21, \beta=M=0.5, \phi=0.1$ and $\gamma=1$ 
The effect of magnetic parameter $M$ on surface temperature $\theta(0)$ is illustrated in Figure 3 . Form figure, it is clearly shown that the increase of $M$ enhanced the values of $\theta(0)$. Further, from numerical calculation, the blood-based Casson ferrofluid with 0.1 vol. of ferrite gave at least $20.8 \%$ enhancement in $\theta(0)$ compared to the blood-based fluid. Ferrofluid with 0.2 vol. ferroparticles score better with $46 \%$ increment in $\theta(0)$. The positive increment increases as $M$ increases.

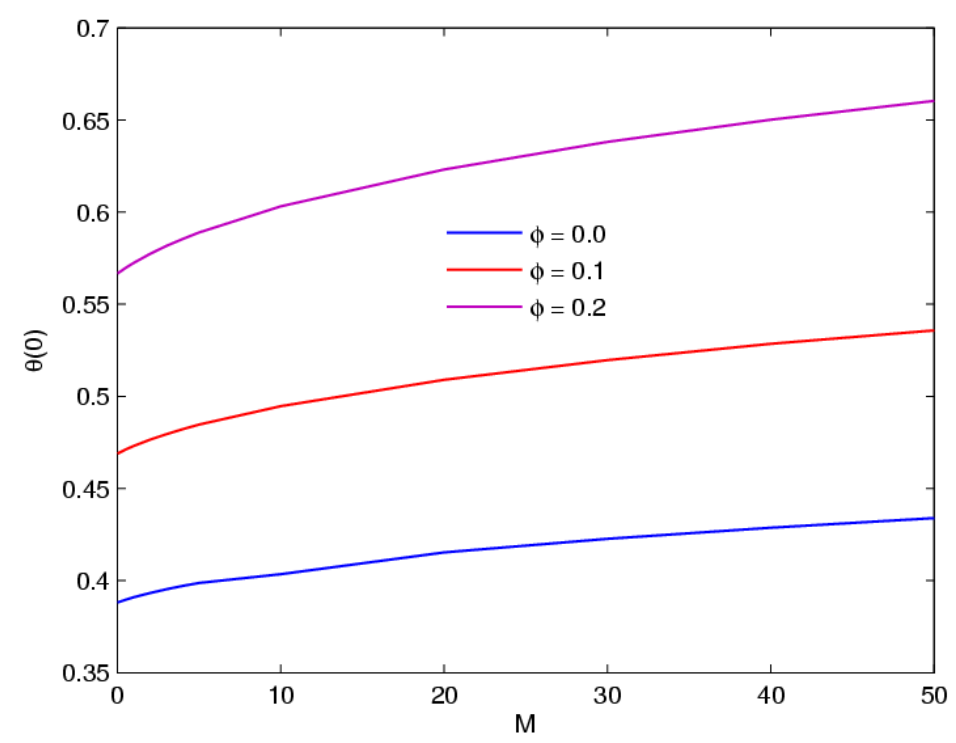

Fig. 3. Variation of $\theta(0)$ with various values of $M$ when $\operatorname{Pr}=21, \beta=\varepsilon=0.5$ and $\gamma=1$

In discussing the fluid friction with stretching surface, Figure 4 and 5 are illustrated. From Figure 4 , it is found that the values of the skin friction coefficient $C_{f} \operatorname{Re}_{x}^{1 / 2}$ is negative for $\varepsilon<1$, due to the fact that the stretching surface and fluid move in an opposite direction. As $\varepsilon=1$, the values of $C_{f} \operatorname{Re}_{x}^{1 / 2}=0$, as the stretching plate and a fluid have the same velocity and direction. Next, it is found that the increase of $\varepsilon$ for $\varepsilon>1$ results to the increase in $C_{f} \operatorname{Re}_{x}^{1 / 2}$.

This is realistic since the increase of $\varepsilon$ implies to the increase of ratio for free stream velocity over a stretching velocity. The velocity differences increase thus reflect to the increase in $C_{f} \operatorname{Re}_{x}^{1 / 2}$. Next, it is suggested that the values of $C_{f} \mathrm{Re}_{x}^{1 / 2}$ for $\mathrm{Fe}_{3} \mathrm{O}_{4}$ blood-based Casson ferrofluid and cobalt ferrite $\mathrm{CoFe}_{2} \mathrm{O}_{4}$ blood-based Casson ferrofluid gave closed results. The present of ferroparticles in ferrofluid influence to the increase in $C_{f} \mathrm{Re}_{x}^{1 / 2}$ and the temperature difference between the blood and the blood-based Casson ferrofluid is more significant as $\varepsilon$ increasing.

Figure 5 shows the variation of skin friction coefficient $C_{f} \operatorname{Re}_{x}^{1 / 2}$ against magnetic parameter $M$ with various ferroparticle volume fraction $\phi$. It is observed that the values of $C_{f} \operatorname{Re}_{x}^{1 / 2}$ are negative due to the fact that the opposite direction between stretching plate and the fluid flow. The increase of $M$ results to the increase of these quantities. In considering the effects of ferroparticle volume fraction $\phi$, the blood-based Casson ferrofluid with 0.1 vol of ferroparticles sparks a significant increment of $C_{f} \operatorname{Re}_{x}^{1 / 2}$ compared to the blood-based fluid. Noticed that, doubling the ferroparticles 
$\left(0.2\right.$ vol) shows an only small increment in $C_{f} \mathrm{Re}_{x}^{1 / 2}$. Further, it is worth mentioning that the effects of $\phi$ are more significant as $M$ increases.

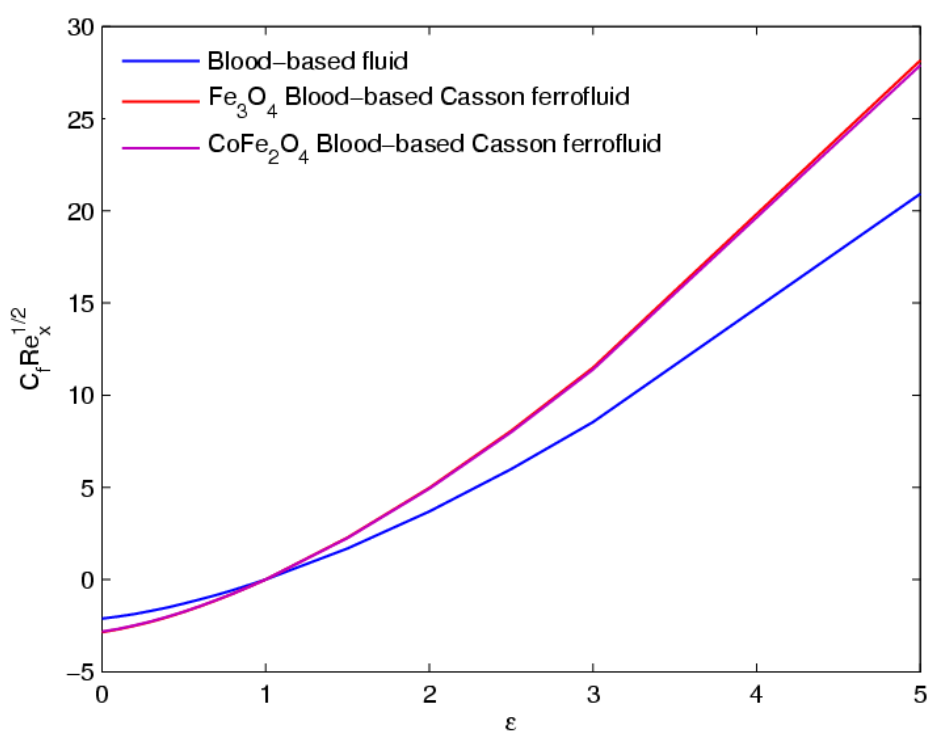

Fig. 4. Variation of $C_{f} \operatorname{Re}_{x}^{1 / 2}$ with various values of $\varepsilon$ when $\operatorname{Pr}=21, \beta=M=0.5, \phi=0.1$ and $\gamma=1$

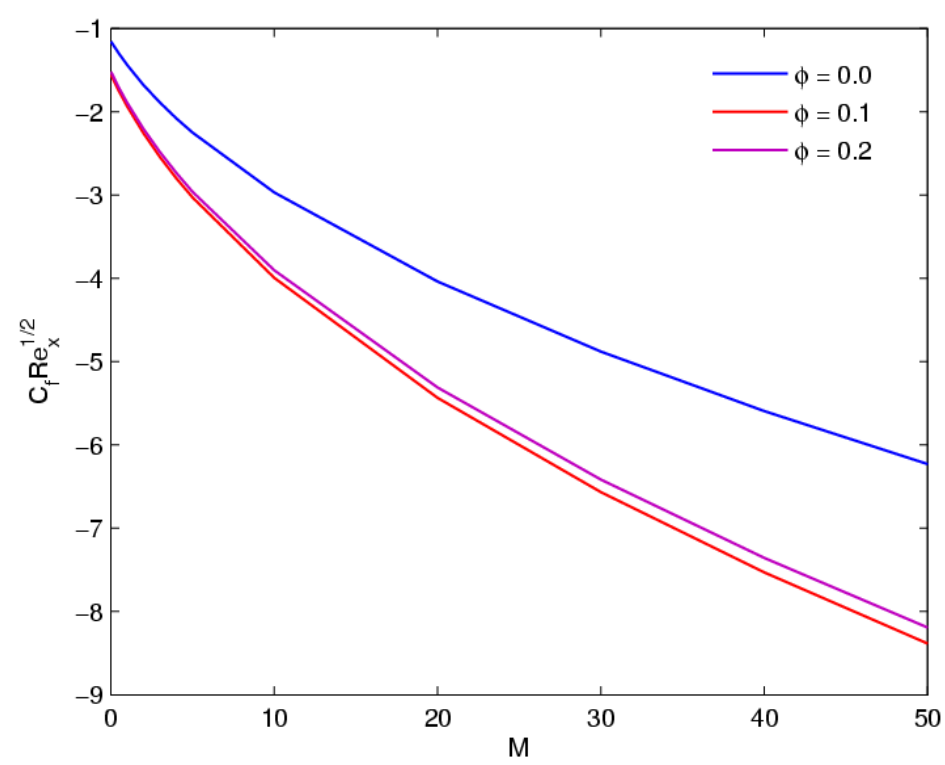

Fig. 5. Variation of $C_{f} \operatorname{Re}_{x}^{1 / 2}$ with various values of $M$ when $\operatorname{Pr}=21, \beta=\varepsilon=0.5$ and $\gamma=1$

Next, it is worth to investigates effects of fluid parameter on the temperature and the velocity distribution across the boundary layer as well as its thicknesses. Figure 6 and 7 illustrate the temperature profiles $\theta(\eta)$ for various values of $\beta$ and $\phi$, respectively. From Figure 6 , it is concluded that the increase in $\beta$ results to a small increment in temperature and the thermal boundary layer thicknesses. Physically, this shows that the rheological behaviour in blood-based Casson ferrofluid contribute less effects on the heat transfer process and nearly similar to Newtonian-blood based ferrofluid $(\beta \rightarrow \infty)$. 
In Figure 7, the increase in ferroparticle volume fraction gave a significant increasing in temperature as well as the thickness of the thermal boundary layer. Since the heat transfer coefficient is directly proportional to the temperature Eq. (10), physically the increase of ferroparticle composition in blood-based Casson ferrofluid results to the enhancement to this quantity. It is worth to limit the ferroparticle volume fraction to a small composition (i.e; $10 \%$ ) in order to prevent clogging, maintaining the stability and viscosity of a Casson ferrofluid.

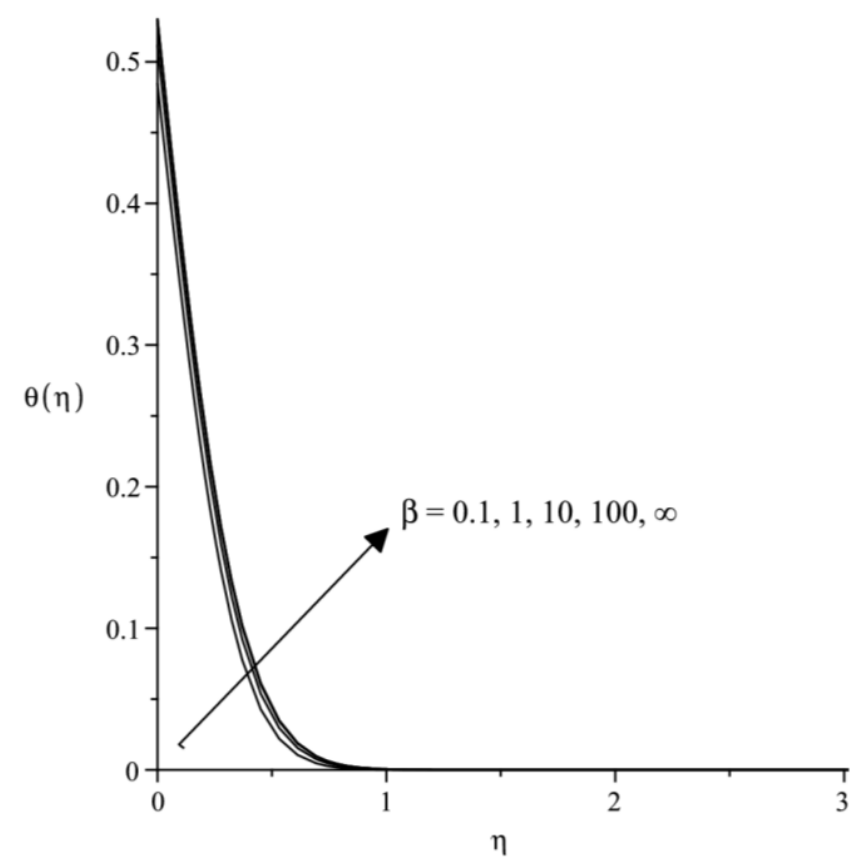

Fig. 6. Temperature profiles $\theta(\eta)$ with various values of $\beta$ when $\operatorname{Pr}=21, \phi=0.1, M=\varepsilon=0.5$ and $\gamma=1$

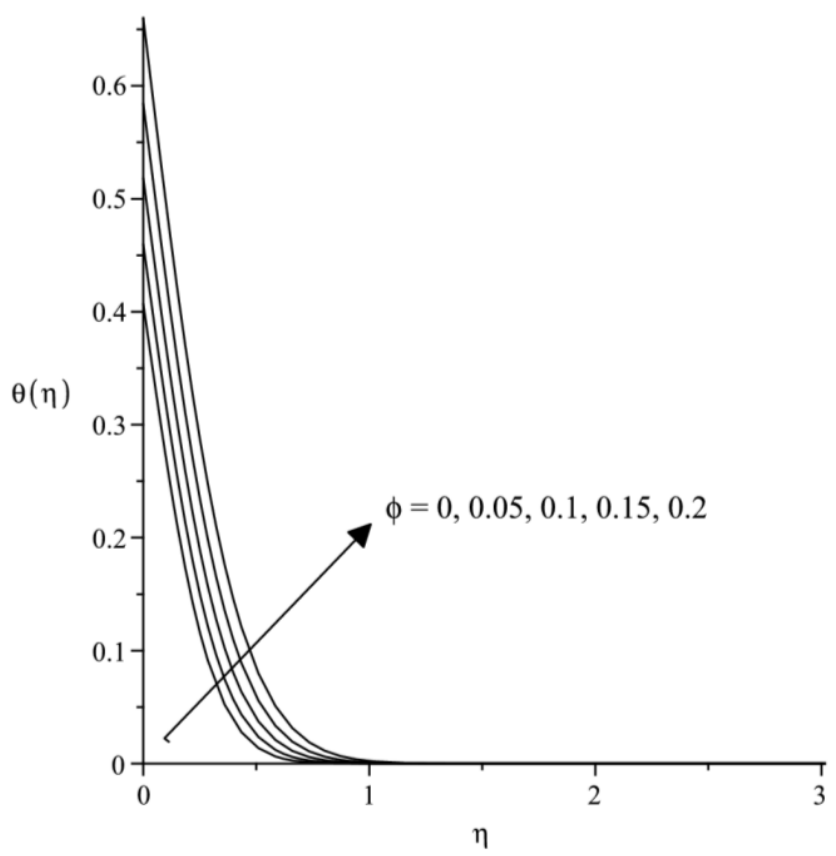

Fig. 7. Temperature profiles $\theta(\eta)$ with various values of $\phi$ when $\operatorname{Pr}=21, \beta=M=\varepsilon=0.5$ and $\gamma=1$ 
Lastly, the velocity profiles for various values of $\beta$ and $\phi$ are shown in Figure 8 and 9, respectively. The scenario for both parameters are contradict with the temperature profiles obtained in Figure 6 and 7. From Figure 8, it is suggested that the increase of Casson parameter results to a drastically reducing in velocity boundary layer thicknesses. Physically, from this pattern, it is shows that the blood-based Casson ferrofluid have a thicker velocity boundary layer compared to a Newtonian-blood based ferrofluid $(\beta \rightarrow \infty)$.

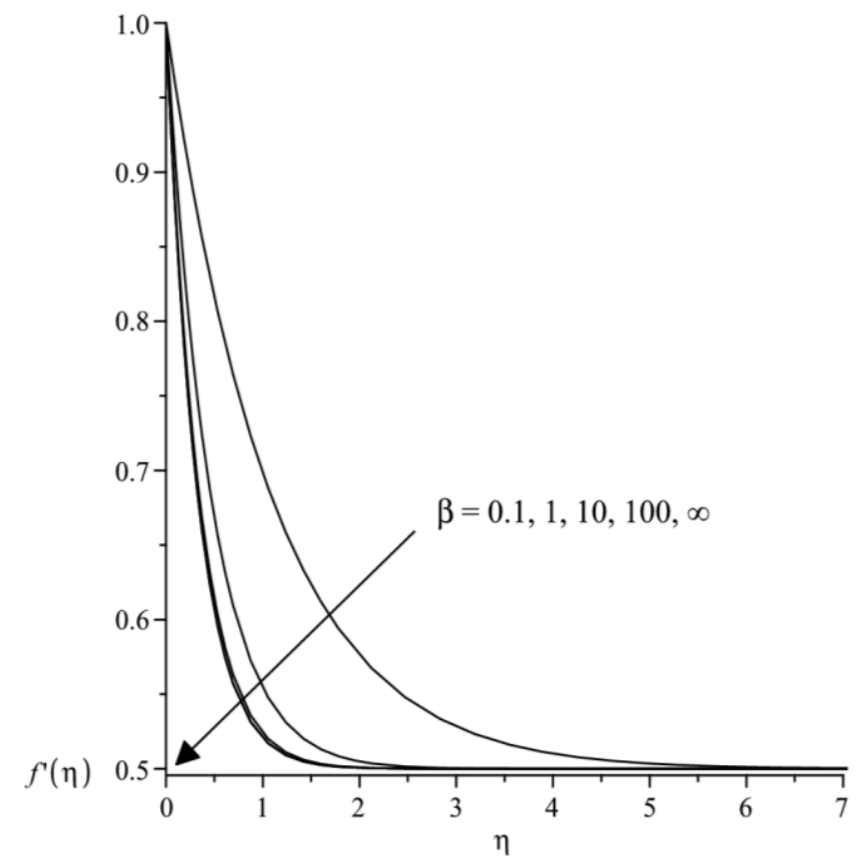

Fig. 8. Velocity profiles $f^{\prime}(\eta)$ with various values of $\beta$ when $\operatorname{Pr}=21, \phi=0.1, M=\varepsilon=0.5$ and $\gamma=1$

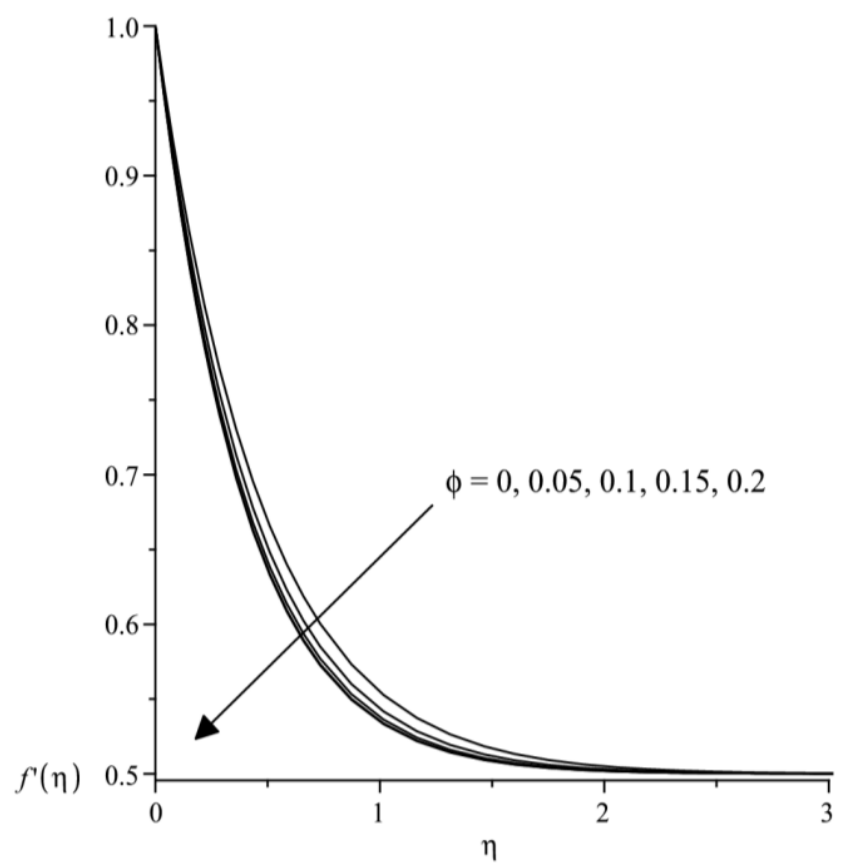

Fig. 9. Velocity profiles $f^{\prime}(\eta)$ with various values of $\phi$ when $\operatorname{Pr}=21, \beta=M=\varepsilon=0.5$ and $\gamma=1$ 
Further, the increase of velocity boundary layer thickness on blood-based Casson ferrofluid is the sign of the velocity gradient reduction thus suggested the blood-based Casson ferrofluid have low in skin friction coefficient compared to a Newtonian-blood based ferrofluid. On the other hand, the increase in ferroparticle composition in blood-based Casson ferrofluid had slightly reduced the velocity boundary layer thicknesses. The increase of ferroparticle volume fraction physically increase the fluid density thus results to the enhancing in skin friction coefficient. This situation can be determined clearly from the increase in velocity gradient in Figure 9.

\section{Conclusions}

The MHD stagnation point flow of a blood-based Casson ferrofluid on a stretching sheet with Newtonian heating was numerically studied. It was shown how the stretching parameter $\varepsilon$, magnetic parameter $M$ and the ferroparticle volume fraction $\phi$ in blood-based Casson ferrofluid affect the surface temperature $\theta(0)$ and the skin friction coefficient $C_{f} \mathrm{Re}_{x}^{1 / 2}$.

As a summary, in the absence of stretching effects, it is found that surface temperature for cobalt and ferrite blood-based Casson ferrofluid provided $17.1 \%$ and $21.4 \%$ higher than the bloodbased fluid. The presence of stretching parameter reduced this difference while increase the skin friction coefficient. Next, the increase of $M$ enhanced the values of surface temperature and skin friction coefficient.

Further, it is concluded that the blood-based Casson ferrofluid with 0.1 vol of ferrite gave at least $20.8 \%$ enhancement in surface temperature compared to blood while the 0.2 vol. ferroparticles score better with $46 \%$ increment in surface temperature as magnetic parameter, $M$ presence. Lastly, it is found that the increase of ferroparticle volume fraction gave a significantly increase in heat transfer coefficient. Meanwhile, it is worth mentioning that, from numerical computation, the blood-based Casson ferrofluid contribute lower skin friction coefficient compared to Newtonian-blood based ferrofluid.

\section{Acknowledgement}

Authors gratefully acknowledge the financial and facilities support from the Malaysia Ministry of Education via Fundamental Research Grant Scheme FRGS/1/2019/STG06/UMP/02/1 (RDU1901124) and Universiti Malaysia Pahang.

\section{References}

[1] Hayat, T., S. A. Shehzad, A. Alsaedi, and M. S. Alhothuali. "Mixed convection stagnation point flow of Casson fluid with convective boundary conditions." Chinese Physics Letters 29, no. 11 (2012): 114704. https://doi.org/10.1088/0256-307X/29/11/114704

[2] Crane, Lawrence J. "Flow past a stretching plate." Zeitschrift für angewandte Mathematik und Physik ZAMP 21, no. 4 (1970): 645-647. https://doi.org/10.1007/BF01587695

[3] Malvandi, A., F. Hedayati, and D. D. Ganji. "Slip effects on unsteady stagnation point flow of a nanofluid over a stretching sheet." Powder Technology 253 (2014): 377-384. https://doi.org/10.1016/j.powtec.2013.11.049

[4] Mohamed, Muhammad Khairul Anuar, Muhammad Imran Anwar, Sharidan Shafie, Mohd Zuki Salleh, and Anuar Ishak. "Effects of Magnetohydrodynamic on the Stagnation Point Flow past a Stretching Sheet in the Presence of Thermal Radiation with Newtonian Heating." In International Conference on Mathematical Sciences and Statistics 2013, pp. 155-163. Springer, Singapore, 2014. https://doi.org/10.1007/978-981-4585-33-0 16

[5] Ramanaiah, G. V., Babu, M. S., and Lavanya, M. "Mhd Mixed Convection Stagnation-Point Flow of a Power-Law Nanofluid Towards a Stretching Surface in the Presence of Viscous Dissipation and Suction/Injection." Imperial Journal of Interdisciplinary Research 2, no. 4 (2016): 56-74.

[6] Soid, S. K., Ishak, A., and Pop, I. "Mhd Stagnation-Point Flow over a Stretching/Shrinking Sheet in a Micropolar Fluid with a Slip Boundary." Sains Malaysiana 47, no. 11 (2018): 2907-2916. https://doi.org/10.17576/jsm-2018$\underline{4711-34}$ 
[7] Mohamed, Muhammad Khairul Anuar, Nor Aida Zuraimi Md Noar, Zulkhibri Ismail, Abdul Rahman Mohd Kasim, Norhafizah Md Sarif, Mohd Zuki Salleh, and Anuar Ishak. "Slip effect on stagnation point flow past a stretching surface with the presence of heat generation/absorption and Newtonian heating." In AIP Conference Proceedings, vol. 1867, no. 1, p. 020009. AIP Publishing LLC, 2017. https://doi.org/10.1063/1.4994412

[8] Mabood, F., Shateyi, S., Rashidi, M. M., Momoniat, E., and Freidoonimehr, N. "Mhd Stagnation Point Flow Heat and Mass Transfer of Nanofluids in Porous Medium with Radiation, Viscous Dissipation and Chemical Reaction." Advanced Powder Technology 27, no. 2 (2016): 742-749. https://doi.org/10.1016/i.apt.2016.02.033

[9] Hsiao, K.-L. "Stagnation Electrical Mhd Nanofluid Mixed Convection with Slip Boundary on a Stretching Sheet." Applied Thermal Engineering 98 (2016): 850-861. https://doi.org/10.1016/i.applthermaleng.2015.12.138

[10] Mohamed, M. K. A., Salleh, M. Z., Ishak, A., and Pop, I. "Stagnation Point Flow and Heat Transfer over a Stretching/Shrinking Sheet in a Viscoelastic Fluid with Convective Boundary Condition and Partial Slip Velocity." The European Physical Journal Plus 130, no. 8 (2015): 1-9. https://doi.org/10.1140/epjp/i2015-15171-8

[11] Hayat, T., Asad, S., Mustafa, M., and Alsaedi, A. "Mhd Stagnation-Point Flow of Jeffrey Fluid over a Convectively Heated Stretching Sheet." Computers \& $\quad$ Fluids $108 \quad$ (2015): $179-85$. https://doi.org/10.1016/i.compfluid.2014.11.016

[12] Mustafa, Meraj, Tasawar Hayat, Pop loan, and Awatif Hendi. "Stagnation-point flow and heat transfer of a Casson fluid towards a stretching sheet." Zeitschrift für Naturforschung A 67, no. 1-2 (2012): 70-76. https://doi.org/10.5560/zna.2011-0057

[13] Yasin, S. H. M., Mohamed, M. K. A., Ismail, Z., and Salleh, M. Z. "Mathematical Solution on Mhd Stagnation Point Flow of Ferrofluid." Defect and Diffusion Forum $399 \quad$ (2020): 38-54. https://doi.org/10.4028/www.scientific.net/DDF.399.38

[14] Anuar, Nur Syazana, Norfifah Bachok, Norihan Md Arifin, and Haliza Rosali. "Effect of Suction/Injection on Stagnation Point Flow of Hybrid Nanofluid over an Exponentially Shrinking Sheet with Stability Analysis." CDF Letters 11, no. 12 (2019): 21-33. https://doi.org/10.3390/sym11040522

[15] Makanda, G., Shaw, S., and Sibanda, P. "Effects of Radiation on Mhd Free Convection of a Casson Fluid from a Horizontal Circular Cylinder with Partial Slip in Non-Darcy Porous Medium with Viscous Dissipation." Boundary Value Problems 2015, no. 1 (2015): 1-14. https://doi.org/10.1186/s13661-015-0333-5

[16] Khalid, Asma, Ilyas Khan, Arshad Khan, Sharidan Shafie, and I. Tlili. "Case study of MHD blood flow in a porous medium with CNTS and thermal analysis." Case studies in thermal engineering 12 (2018): 374-380. https://doi.org/10.1016/i.csite.2018.04.004

[17] Rashad, A. M. "Impact of Anisotropic Slip on Transient Three Dimensional Mhd Flow of Ferrofluid over an Inclined Radiate Stretching Surface." Journal of the Egyptian Mathematical Society 25, no. 2 (2017): $230-37$. https://doi.org/10.1016/j.joems.2016.12.001

[18] Stephen, Papell Solomon. "Low viscosity magnetic fluid obtained by the colloidal suspension of magnetic particles." U.S. Patent 3,215,572, issued November 2, 1965.

[19] Zeeshan, A., Majeed, A., and Ellahi, R. "Effect of Magnetic Dipole on Viscous Ferro-Fluid Past a Stretching Surface with Thermal Radiation." Journal of Molecular liquids $215 \quad$ (2016): 549-54. https://doi.org/10.1016/j.molliq.2015.12.110

[20] Das, Sarit K., Stephen U. Choi, Wenhua Yu, and T. Pradeep. Nanofluids: science and technology. John Wiley \& Sons, 2007. https://doi.org/10.1002/9780470180693

[21] Mohamed, M. K. A., Salleh, M. Z., Nazar, R., and Ishak, A. "Stagnation Point Flow over a Stretching Sheet with Newtonian Heating." Sains Malaysiana 41, no. 11 (2012): 1467-73. https://doi.org/10.1063/1.4757492

[22] Merkin, J. H. "Natural-Convection Boundary-Layer Flow on a Vertical Surface with Newtonian Heating." International Journal of Heat and Fluid Flow 15, no. 5 (1994): 392-98. https://doi.org/10.1016/0142727X(94)90053-1

[23] Mohamed, Muhammad Khairul Anuar, Nurul Ainn Ismail, Norhamizah Hashim, Norlianah Mohd Shah, and Mohd Zuki Salleh. "MHD Slip Flow and Heat Transfer on Stagnation Point of a Magnetite (Fe3O4) Ferrofluid towards a Stretching Sheet with Newtonian Heating." CFD Letters 11, no. 1 (2019): 17-27.

[24] Bhattacharyya, K. "Mhd Stagnation-Point Flow of Casson Fluid and Heat Transfer over a Stretching Sheet with Thermal Radiation." Journal of Thermodynamics 2013 (2013): 1-9. https://doi.org/10.1155/2013/169674

[25] Nazar, R., Amin, N., Filip, D., and Pop, I. "Unsteady Boundary Layer Flow in the Region of the Stagnation Point on a Stretching Sheet." International Journal of Engineering Science 42, no. 11 (2004): 1241-53. https://doi.org/10.1016/i.ijengsci.2003.12.002 